\title{
EVALUATION OF SELECTED FEATURES FOR CAR DETECTION IN AERIAL IMAGES
}

\author{
Sebastian Tuermer ${ }^{\mathrm{a}^{*}}$, Jens Leitloff $^{\mathrm{a}}$, Peter Reinartz ${ }^{\mathrm{a}}$, Uwe Stilla $^{\mathrm{b}}$ \\ ${ }^{a}$ Remote Sensing Technology Institute (IMF), German Aerospace Center (DLR) \\ 82234 Oberpfaffenhofen, Germany \\ sebastian.tuermer@dlr.de,jens.leitloff@dlr.de,peter.reinartz@dlr.de, \\ ${ }^{\mathrm{b}}$ Photogrammetry and Remote Sensing (PRS), Technische Universitaet Muenchen (TUM) \\ Arcisstrasse 21, 80333 Munich, Germany \\ stilla@tum.de
}

Commission III/4

KEY WORDS: vehicle detection, aerial images, Haar-like features, HoG features, Boosting, 3K camera system

\begin{abstract}
:
The extraction of vehicles from aerial images provides a wide area traffic situation within a short time. Applications for the gathered data are various and reach from smart routing in the case of congestions to usability validation of roads in the case of disasters. The challenge of the vehicle detection task is finding adequate features which are capable to separate cars from other objects; especially those that look similar. We present an experiment where selected features show their ability of car detection. Precisely, Haar-like and HoG features are utilized and passed to the AdaBoost algorithm for calculating the final detector. Afterwards the classifying power of the features is accurately analyzed and evaluated. The tests a carried out on aerial data from the inner city of Munich, Germany and include small inner city roads with rooftops close by which raise the complexity factor.
\end{abstract}

\section{INTRODUCTION}

The improvements of advanced driver assistance systems achieved in the last decades are impressive; individual mobility has never been more comfortable as today. Modern cars are littered with helpful gadgets. Just think on systems like parking sensors, rain sensor, adaptive light control or active speed control, to name a few of the latest operational inventions. And of course not to forget navigation systems which are capable of routing you directly to your destination without the necessity of reading a map. No doubt all of these assistant systems make life remarkable easier. However all of these technical accomplishments are not worth anything if the roads are congested and driving is not possible.

Due to this aspect a lot of research is done developing traffic surveillance systems (Meffert et al., 2005) which should indicate traffic jams and provide alternative routing in addition. Groundwork of all the routing attempts is the detection of the cars. Nowadays the information about the position of the cars is mainly received by induction loops, car to car communication, floating car solutions or stationary video cameras. A novel approach which is in its infancy takes the position of the navigation system (GPS coordinates) and sends the information to a data acquisition center. Current research is carried out by Tomtom a Dutch manufacture of navigation systems. They do not want to introduce a new transmitting module but take the driver's mobile phone. That takes us to another innovative method, Observed Time Difference of Arrival (OTDOA) which is only working in UMTS environment and delivers positioning data with an accuracy ranging from 50 to 100 meters. However all of the methods mentioned are not able to satisfy our needs completely.

The acquisition method that fits best to our prerequisites is aerial imaging. The most important advantages are rapid availability, high positional accuracy and the capability of covering large areas within less time. The system is mainly developed to get realtime traffic information in the case of mass events or catastrophes. But by far this is not the only application, also traffic analysts could benefit by using the data weeks later for validating their road planning including traffic signals and speed limits. And not to forget a further advantage, the by-product real-time mapping which could show broken or blocked roads after natural disasters.

Finally, to obtain the traffic data automatically a wide variety of methods for car extraction from aerial imagery can be consulted. Some are explicit trying to find a predefined model in the search image whereas others use implicit methods where the model is created by example images. But all of them have a similar challenge which is finding features that describe the object optimally.

We contribute to the workshop a detailed testing and evaluation of selected features for car detection. These features are Haarlike and HoG features. All of these features run through the same machine learning process. This should ensure the comparability of returned results. The AdaBoost algorithm calculates the final classifier for each feature set. Afterwards, the classification capability of each detector is determined. We take prominent key figures like recall or precision rate for the comparison process. But also facts like computation time or expandability come into account. The test dataset consists of aerial images with a resolution of approximately $15 \mathrm{~cm}$ from a professional off the shelf digital frame camera. Test area is the inner city of Munich with its small roads and high buildings.

\section{RELATED WORK}

A feasible way of classifying methods for vehicle detection in optical images, is splitting in three groups according to the platform of the sensor. The field with definitely the highest amount of research activity during the last years are stationary video cameras which provide side view images or at least oblique view images. Further property is a quite high imaging frequency in comparison to the other groups. The use of wavelet coefficients as features and AdaBoost can be seen in (Schneiderman and Kanade, 2000). Also (She et al., 2004) are detecting cars by the use of Haar wavelets features in the HSV color space. Utilizing color information is the way of (Knauer et al., 2005) as well. They use 
multi dimensional color histograms. A combination of Haar and HoG features which are formed to a strong cascading classifier by Boosting presents (Negri et al., 2008). In (Kasturi et al., 2009) a simple background subtraction is done which is only working for video data. An overview on the work for stationary cameras can be found in (Sun et al., 2006).

The next group considers satellite imagery which provide a reduced spatial resolution (highest resolution is often max $0.5 \mathrm{~m}$ ) and mainly use single images, not time series. An approach which uses simple features based on shape and intensity presents (Eikvil et al., 2009). Using segmented images and apply a maximum likelihood classification can be observed in (Larsen et al., 2009). Promising results have also been achieved by (Leitloff et al., 2010). They use Haar-like features in combination with AdaBoost.

The last group of approaches deals with airborne images. At this step we first suggest a further separation in explicit or implicit models. Approaches based on explicit models are for example given in (Moon et al., 2002) with a convolution of a rectangular mask and the original image. Also (Zhao and Nevatia, 2003) offer an interesting method by creating a wire-frame model and try to match it with extracted edges at the end of a Bayesian network. A similar way is suggested by (Hinz, 2003a) (Hinz, 2003b), the author makes the approach more mature and added additional parameters like the position of the sun. Another proposed method of (Lenhart et al., 2008) uses a sophisticated blob detection. Color information is used as well as previous knowledge of the travel direction. (Kozempel and Reulke, 2009) provide a very fast solution which takes four special shaped edge filters trying to represent an average car. Finally implicit modeling is used by (Grabner et al., 2008), they take Haar-like features, HoG features and LBP (local binary patterns). All these features are passed to an on-line AdaBoost training algorithm which creates a strong classifier.

A comprehensive overview and evaluation of airborne sensors for traffic estimation can be found in (Hinz et al., 2006) and (Stilla et al., 2004).

\section{THEORETICAL BACKGROUND}

The following section enlightens briefly the utilized methods or algorithms for this experiment. The experimental setting is shown in Fig. 1. Main focus is on evaluating the effectiveness of the different composed detectors.

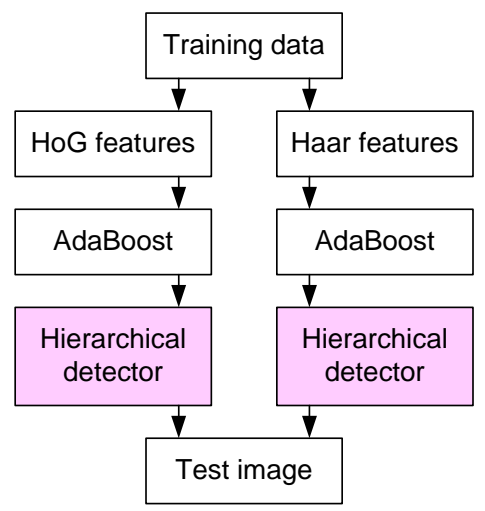

Figure 1: Experimental setting

\subsection{Boosting}

Boosting is the training method to obtain our vehicle detector. The general idea of boosting is the creation of a strong classifier by combining several weak classifiers. Where weak classifiers are classifiers that are better than chance. At first this has been done by (Schapire, 1990) (Freund, 1990) but the method was not adaptive at this time. Due to this missing characteristic, variants like AdaBoost have been developed (Freund and Schapire, 1997).

\subsection{Haar-like features}

Haar wavelets are functions calculating the difference of intensities. A first approach using this features was presented by (Papageorgiou et al., 1998). Soon, a short time later (Viola and Jones, 2001) took up this idea and proposed the so called Haar-like features. The functions are applied on different sized regions and different positions in the detection window. We utilize the reduced original feature set represented in Fig. 2. Where the white field is subtracted of the black one. One of the most important ad-

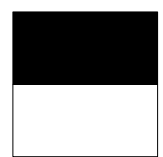

(1)

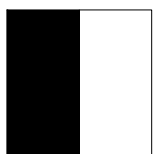

(2)

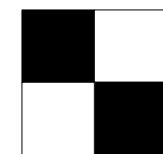

(3)

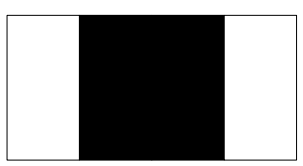

(4)

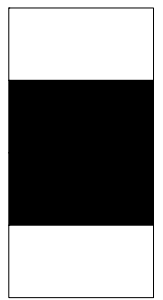

(5)
Figure 2: Haar-like features

vantages over a lot of competitive features is the rapid processing time due to integral images. The integral image has to be calculated just once and enables a fast computation of all Haar-like features.

\subsection{HoG features}

Originally, HoG features were introduced by (Dalal and Triggs, 2005). We decide to chose this kind of feature due to its proven ability of describing objects simply and efficiently (Zhu et al., 2006). To speed up the calculation process integral histograms can be used (Porikli, 2005), similar to integral images in Subsection 3.2 Haar-like features. The creation process starts by sliding a window over a gradient image. Every window contains certain sub-windows which are slided over the whole area of the window. Now the features are created by quantize gradient magnitudes from every sub-window to a histogram. The particular bin is chosen according to the gradient orientation. The schema in Fig. 3 shows the process of origin. A detailed explanation of these features and how the feature extraction works can be found in (Tuermer et al., 2010).

\subsection{Multi-detection suppression}

Unfortunately, the proposed car detection method is prone to multi detections. There are several ways to avoid the undesired effect. One could be limiting the examination of the search image to every second or third pixel. However some vehicles will be unrecognized due to that method. Therefor we decided to introduce 


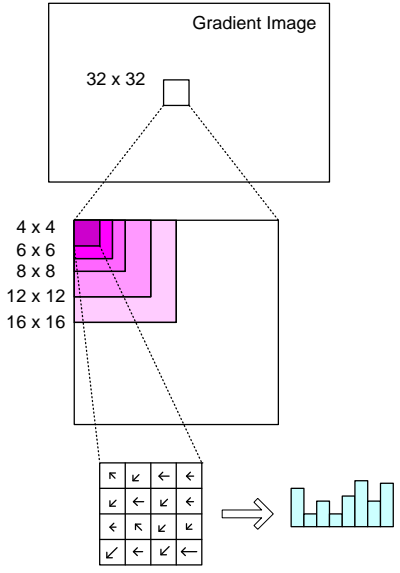

Figure 3: Steps of creating a HoG feature

a technique which uses the confidence value returned by the application of the detector and the distance of the single detections. Inertial point is that cars must have a certain space between each other. The idea is realized by shifting a rectangle having the average size of a car over the confidence image. Only the detection of the highest value remains. The technique is illustrated in Fig. 4.

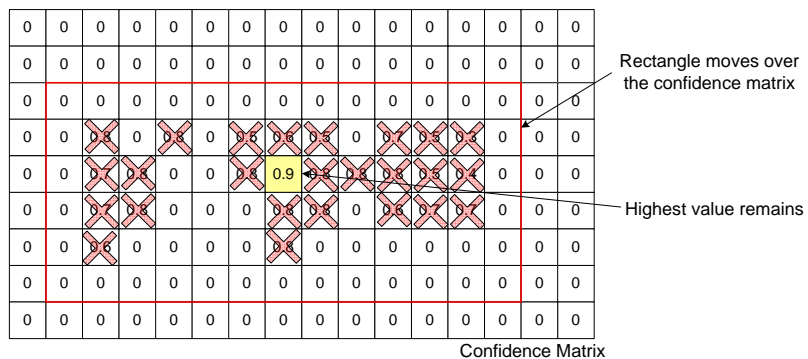

Figure 4: Suppression of multi detection

\section{RESULTS AND DISCUSSION}

The aerial image used for this test has been taken by the DLR $3 \mathrm{~K}$ camera system and has $15 \mathrm{~cm}$ spatial resolution. More information about the sensor can be found in (Tuermer et al., 2010). The road in the image is located close to the Technical University of Munich which is surrounded by high buildings with a lot of dormers. Especially dormers are often miss-classified due to its car-like shape.

\subsection{Results}

The three different detectors are applied to every pixel position of the test image. Usually road databases would be used to exclude areas where cars appear unlikely. But common road databases (e.g. Navteq) have a poor accuracy and therefore applying the detector on the roofs beside the road is not an unrealistic scenario. Hence we do not use additional ways of limiting the search space. Further remark is that only cars in the north-south direction and vice versa are the aim of the detection. A reason is the reduced training database of positive vehicles and the method itself which is not rotation invariant up to now.

The experimental results are partitioned in the following schema. The image we present first of each utilized feature set has no further processing steps. It shows a lot of false and multi detections. Whereas the second image is treated with the multi detection suppression procedure and a certain threshold.
The cascading detector composed of the Haar-like features has seven hierarchical levels. Generally it can be assumed that a higher level implicates a higher amount of features. As depicted in Tab. 1, the first level uses three features whereas the last level consists of 13 linearly weighted features. The features are chosen from a pool of 11960 different ones. They are of size $1 \times 1,2 \times 2$, $4 \times 4$ and $8 \times 8$ pixels (for the features (1), (2), (3) in Fig. 2). The feature (4) is used with size $1 \times 2,2 \times 4,4 \times 8$ and $8 \times 16$; inversely arranged in the case of feature (5). Applying the detector to the test image results in Fig. 5 (a) and after the post-processing Fig. 5 (b).

\begin{tabular}{cccccccc}
\hline hierarchical level & 1 & 2 & 3 & 4 & 5 & 6 & 7 \\
\hline Haar-like features & 3 & 4 & 5 & 6 & 8 & 10 & 13
\end{tabular}

Table 1: Distribution of features used in the hierarchical detector with Haar-like features

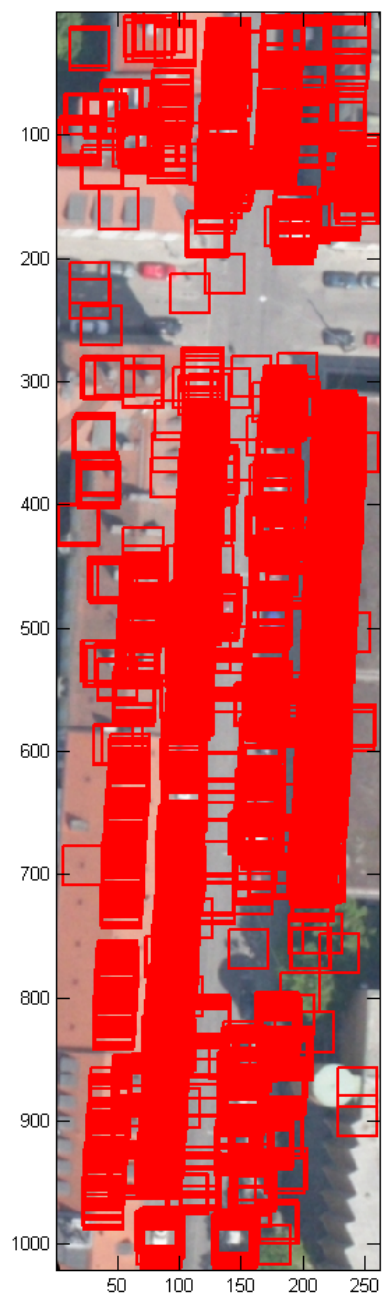

(a) without post-processing

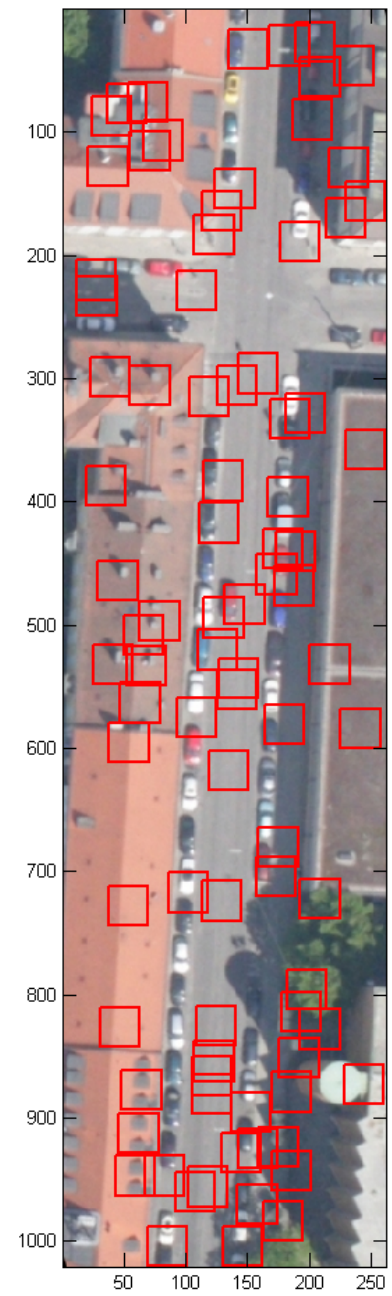

(b) with threshold and multi detection suppression
Figure 5: Result Haar-like features

The pure HoG based detector utilizes features of size $4 \times 4,6 \times 6$, $8 \times 8,12 \times 12$ and $16 \times 16$ pixels as depicted in the sketch of Fig. 3. Summing up the different sized features gives us an overall amount of 2692 features. The detector needs less hierarchical levels as the detector based one Haar-like features and is able to classify in only 5 steps (detailed view of the detector and its levels in Tab. 2). With three features in the first level and seven in the final level. Returned results are in Fig. 6(a) and the reassessed version can be observed in Fig. 6(b). 


\begin{tabular}{cccccc}
\hline hierarchical level & 1 & 2 & 3 & 4 & 5 \\
\hline HoG features & 3 & 4 & 5 & 6 & 7 \\
\hline
\end{tabular}

Table 2: Distribution of features used in the hierarchical detector with HoG features

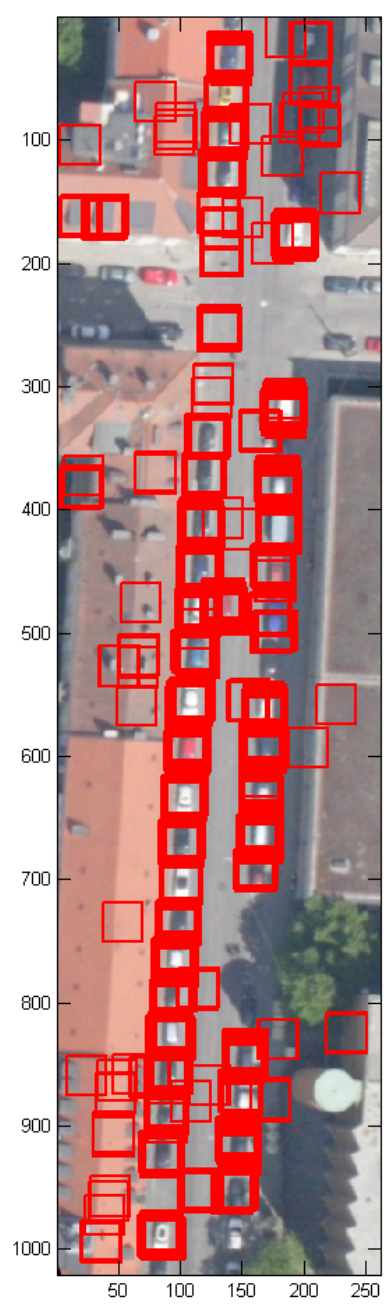

(a) without post-processing

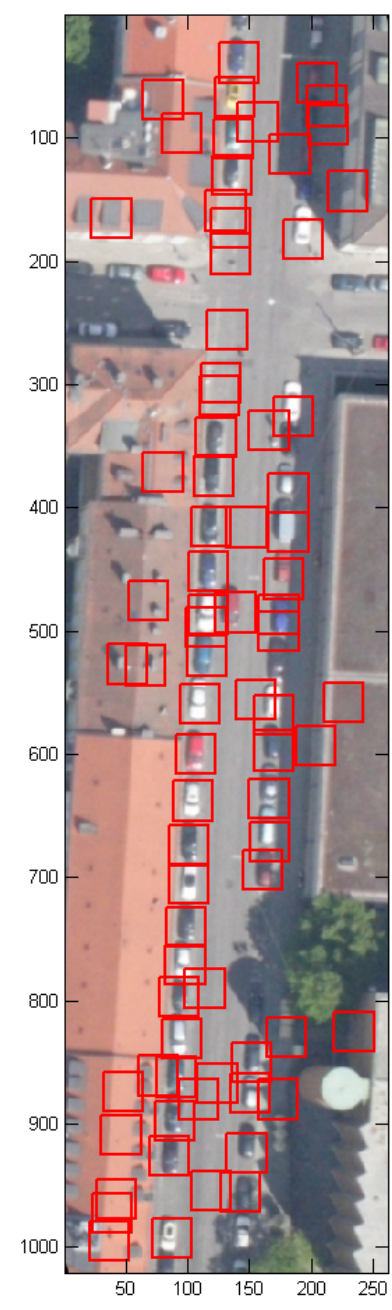

(b) with threshold and multi detection suppression
Figure 6: Result HoG features

Finally the combination of Haar-like and HoG features leads to the results in Fig. 7. The cascading detector has 10 hierarchical levels wherein the two steps at the beginning are composed of HoG features only. In general, the amount of used Haar-like features rises while reaching higher hierarchical levels. The distribution of the features is shown detailed in Fig. 3.

At last prominent statistical measures of the performance of the binary classification can be found in Tab. 4 . Where correctness (also known as precision rate) is defined in Eq. 1, completeness (also recall rate) in Eq. 2 and the definition of quality (accuracy) is expressed in Eq. 3. With abbreviation $T P$ as number of true positives, $F P$ as number of false positives and $F N$ as number of false negatives.

$$
\begin{aligned}
\text { correctness } & =\frac{T P}{T P+F P} \\
\text { completeness } & =\frac{T P}{T P+F N}
\end{aligned}
$$

\begin{tabular}{ccccccccccc}
\hline $\begin{array}{c}\text { hierarchical } \\
\text { level }\end{array}$ & 1 & 2 & 3 & 4 & 5 & 6 & 7 & 8 & 9 & 10 \\
\cline { 1 - 3 } features & & & & & & & & & & \\
\cline { 1 - 2 } & 0 & 0 & 2 & 3 & 4 & 6 & 6 & 10 & 13 & 16 \\
HoG & 3 & 4 & 3 & 3 & 4 & 4 & 7 & 6 & 6 & 8 \\
total & 3 & 4 & 5 & 6 & 8 & 10 & 13 & 16 & 19 & 24 \\
\hline
\end{tabular}

Table 3: Distribution of features used in the hierarchical detector with Haar-like and HoG features mixed

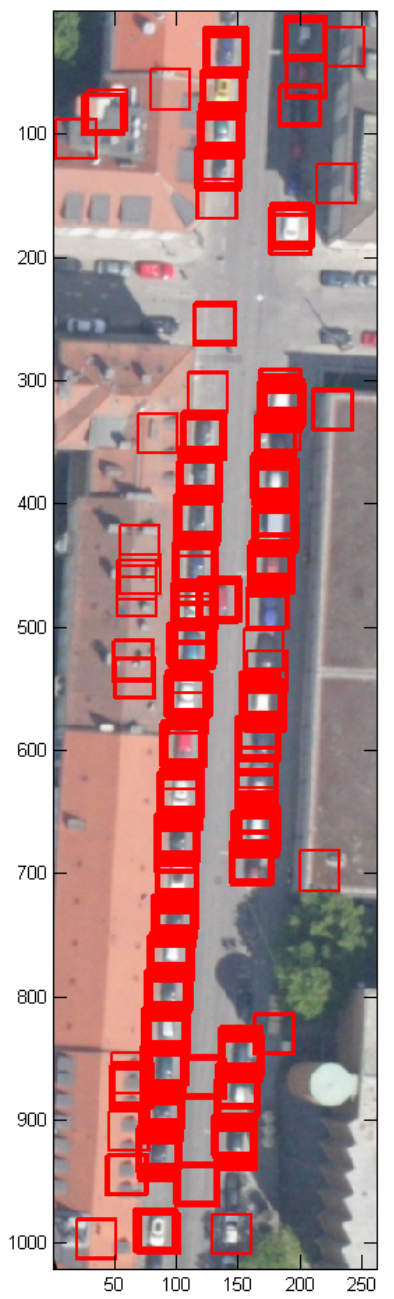

(a) without post-processing

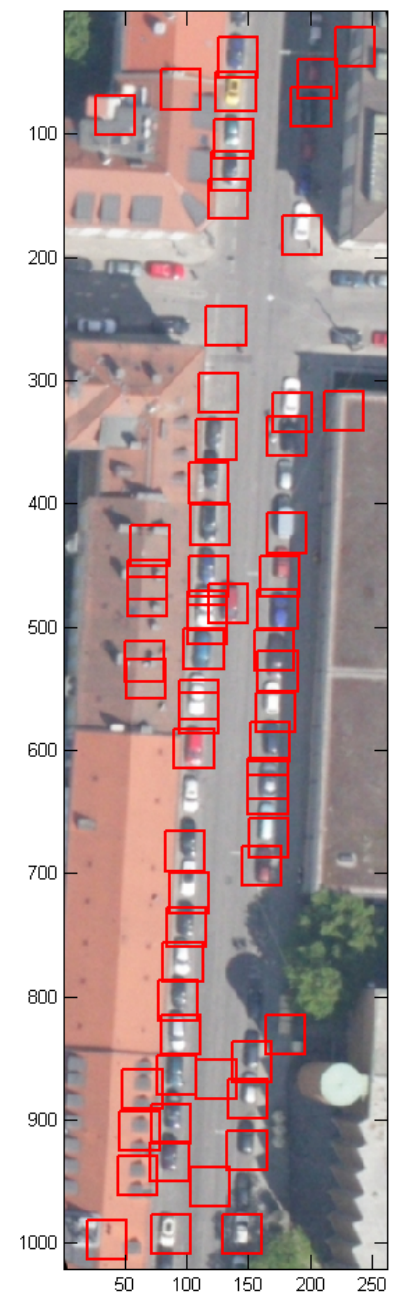

(b) with threshold and multi detection suppression
Figure 7: Result Haar-like and HoG features

$$
\text { quality }=\frac{T P}{T P+F P+F N}
$$

\subsection{Discussion}

While comparing the results of Haar-like (Fig. 5 (a)) and HoG features (Fig. 6(a)) without post-processing, we get the impression at the first glance that the Haar-like based detector delivers much more false and multi detections. But this is innately not a problem because if the true positives have a higher confidence value, the over-classification can be fixed during the postprocessing step. Unfortunately this is not the case, if we have a look at the post-processed detections (Fig. 5 (b) and Fig. 6(b)); the detector only based on Haar-like features failed completely after the multi detection suppression. The HoG features outper- 


\begin{tabular}{|c|c|c|c|}
\hline & Haar & HoG & Haar \& HoG \\
\hline $\begin{array}{l}\text { Detections } \\
\text { (before post-processing) }\end{array}$ & $\overline{7395}$ & $\overline{1231}$ & 1716 \\
\hline $\begin{array}{c}\text { Detections } \\
\text { (after post-processing) }\end{array}$ & 87 & 78 & 61 \\
\hline True positives & 14 & 39 & 40 \\
\hline False positives & 73 & 39 & 21 \\
\hline False negatives & 31 & 8 & 7 \\
\hline Correctness & $16 \%$ & $50 \%$ & $66 \%$ \\
\hline Completeness & $31 \%$ & $83 \%$ & $85 \%$ \\
\hline Quality & $12 \%$ & $45 \%$ & $59 \%$ \\
\hline
\end{tabular}

Table 4: Statistics of detection

form the rival clearly. The primary visual impression can be validated by the figures in Tab. 4 where we juxtapose the correctness, the completeness and the quality rates of the different detectors.

One reasonable explanation for this outcome can be the lack of multifaceted Haar-like features. We ran the tests with the standard feature set (Fig. 2) but there is also an extended version available (published by (Lienhart and Maydt, 2002)) which could enhance the performance. A second assumption is that vehicles which are almost exclusively rectangular could fit better to HoG features in general.

Further interesting fact is that HoG features are used more often in lower hierarchical levels (shown in Tab. 3) whereas Haar-like features appear more often in higher detector levels. This means HoG features prevalently do the coarse classification and Haarlike features are used more often to distinguish between very similar objects.

Finally it is always appreciated to create a fast classifier for real time applications. Usually, a fast detector consists of features which are quickly processed and thereof as less as possible. Haarlike features can be calculated four times faster than four-binHoG features but a lot more Haar-like features are necessary to built a vehicle detector. Additionally, a good deal more detection candidates reach higher detector levels (just look at the final overall detections in Tab. 4 before post-processing). This leads to a three times slower Haar-like based detector than the pure HoG based one.

Also the mixed detector which takes use of both features is slower but after all the detection quality increases.

\section{CONCLUSIONS AND FUTURE WORK}

After finishing the experimental testing of the three different vehicle detectors, we have four concluding fundamental statements.

- A mixture of Haar-like and HoG features increases the vehicle detection quality but takes more calculation time.

- A reduced Haar-like feature set and only AdaBoost is not sufficient for a vehicle detection of high quality.

- HoG features need more calculation time for each single feature (depends on the number of bins) but the detector performs faster due to less features utilized in the detector.

- HoG features show an robust rejection of false positives in early hierarchical levels of the detector; this saves time because remaining detector levels can be skipped.

Our future plan is clearing questions which partly appeared while running the tests for this work. One point is the impact of an extended Haar-like feature set. Our set is just composed of the five most classical features but there are dozens of extensions.

Another point is the introduction of new feature types which could help to make detection more accurate. Also improvements concerning the calculation time are imaginable.

And finally we would like to introduce an optimized post-processing chain which takes all the hypothetical car detections and achieves a deeper examination. The plan is using more information like color or the temporal component. Afterwards another interesting idea could be realized which moves towards a complex probabilistic framework.

\section{REFERENCES}

Dalal, N. and Triggs, B., 2005. Histograms of oriented gradients for human detection. In: Conference on Computer Vision and Pattern Recognition (CVPR) 2005, Vol. 1, IEEE Computer Society, San Diego, CA, USA, pp. 886 - 893.

Eikvil, L., Aurdal, L. and Koren, H., 2009. Classification-based vehicle detection in high-resolution satellite images. ISPRS Journal of Photogrammetry and Remote Sensing 64, pp. 65-72.

Freund, Y., 1990. Boosting a weak learning algorithm by majority. In: Third Annual Workshop on Computational Learning Theory, COLT '90, Morgan Kaufmann Publishers Inc, pp. 202216.

Freund, Y. and Schapire, R. E., 1997. A decision-theoretic generalization of on-line learning and an application to boosting. Journal of Computer and System Sciences 55(1), pp. 119-139.

Grabner, H., Nguyen, T. T., Gruber, B. and Bischof, H., 2008. On-line boosting-based car detection from aerial images. ISPRS Journal of Photogrammetry and Remote Sensing 63(3), pp. $382-$ 396.

Hinz, S., 2003a. Detection and counting of cars in aerial images. In: Proc. International Conference on Image Processing (ICIP), Vol. 2(III), pp. 997-1000.

Hinz, S., 2003b. Integrating local and global features for vehicle detection in high resolution aerial imagery. In: Photogrammetric Image Analysis (PIA), Vol. 34(3), International Archives of Photogrammetry, Remote Sensing and Spatial Information Sciences, pp. 119-124.

Hinz, S., Bamler, R. and Stilla, U., 2006. Editorial theme issue: Airborne und spaceborne traffic monitoring. ISPRS Journal of Photogrammetry and Remote Sensing 61(3-4), pp. 135-136.

Kasturi, R., Goldgof, D., Soundararajan, P., Manohar, V., Garofolo, J., Bowers, R., Boonstra, M., Korzhova, V. and Zhang, J., 2009. Framework for performance evaluation of face, text, and vehicle detection and tracking in video: Data, metrics, and protocol. IEEE Transactions on Pattern Analysis and Machine Intelligence 31(2), pp. 319-336.

Knauer, U., Reulke, R. and Meffert, B., 2005. Fahrzeugdetektion und -erkennung mittels mehrdimensionaler farbhistogrammanalyse. In: Farbbildverarbeitung 2005, Berlin, pp. 93-100.

Kozempel, K. and Reulke, R., 2009. Fast vehicle detection and tracking in aerial image bursts. In: CMRT09, Vol. 38(3/W4), pp. $175-180$.

Larsen, S. O., Koren, H. and Solberg, R., 2009. Traffic monitoring using very high resolution satellite imagery. Photogrammetric Engineering and Remote Sensing 75(7), pp. 859-869.

Leitloff, J., Hinz, S. and Stilla, U., 2010. Vehicle extraction from very high resolution satellite images of city areas. IEEE Trans. on Geoscience and Remote Sensing 48, pp. 1-12. 
Lenhart, D., Hinz, S., Leitloff, J. and U., S., 2008. Automatic traffic monitoring based on aerial image sequences. Pattern Recognition and Image Analysis 18(3), pp. 400 - 405.

Lienhart, R. and Maydt, J., 2002. An extended set of haar-like features for rapid object detection. In: IEEE ICIP 2002, pp. 900 903.

Meffert, B., Blaschek, R., Knauer, U., Reulke, R., Schischmanow, A. and Winkler, F., 2005. Monitoring traffic by optical sensors. In: 2nd International Conference on Intelligent Computing and Information Systems (ICICIS 2005), Cairo, Egypt, pp. 914.

Moon, H., Chellappa, R. and Rosenfeld, A., 2002. Performance analysis of a simple vehicle detection algorithm. Image and Vision Computing 20(1), pp. 1-13.

Negri, P., Clady, X., Hanif, S. M. and Prevost, L., 2008. A cascade of boosted generative and discriminative classifiers for vehicle detection. EURASIP Journal on Advances in Signal Processing 2008, pp. 1-12.

Papageorgiou, C. P., Oren, M. and Poggio, T., 1998. A general framework for object detection. In: Int Computer Vision Conf, pp. 555-562.

Porikli, F., 2005. Integral histogram: a fast way to extract histograms in cartesian spaces. In: Computer Society Conference on Computer Vision and Pattern Recognition (CVPR) 2005, Vol. 1, IEEE Computer Society, San Diego, CA, USA, pp. 829-836.

Schapire, R., 1990. Strength of weak learnability. Machine Learning 5(2), pp. 197-227.

Schneiderman, H. and Kanade, T., 2000. A statistical method for $3 \mathrm{~d}$ object detection applied to faces and cars. In: Conference on Computer Vision and Pattern Recognition (CVPR) 2000, Vol. 1, IEEE Computer Society, pp. 746-751.

She, K., Bebis, G., Gu, H. and Miller, R., 2004. Vehicle tracking using on-line fusion of color and shape features. In: International IEEE Conference on Intelligent Transportation Systems, pp. 731736.

Stilla, U., Michaelsen, E., Soergel, U., Hinz, S. and Ender, J., 2004. Airborne monitoring of vehicle activity in urban areas. In: M. Altan (ed.), International Archives of Photogrammetry, Remote Sensing and Spatial Information Sciences, Vol. 34(B3), pp. 973-979.

Sun, Z., Bebis, G. and Miller, R., 2006. On-road vehicle detection: A review. IEEE Transactions on Pattern Analysis and Machine Intelligence 28(5), pp. 694-711.

Tuermer, S., Leitloff, J., Reinartz, P. and Stilla, U., 2010. Automatic vehicle detection in aerial image sequences of urban areas using $3 \mathrm{~d}$ hog features. In: International Archives of Photogrammetry, Remote Sensing and the Spatial Information Sciences, Vol. XXXVIII(3B), Paris, France, pp. 50-54.

Viola, P. and Jones, M., 2001. Rapid object detection using a boosted cascade of simple features. In: Conference on Computer Vision and Pattern Recognition (CVPR) 2001, Vol. 1, IEEE Computer Society, pp. 511-518.

Zhao, T. and Nevatia, R., 2003. Car detection in low resolution aerial image. Image and Vision Computing 21(8), pp. 693-703.

Zhu, Q., Avidan, S., Yeh, M. and Cheng, K., 2006. Fast human detection using a cascade of histograms of oriented gradients. In: Conference on Computer Vision and Pattern Recognition (CVPR) 2006, Vol. 2, IEEE Computer Society, pp. 14911498. 